infiltration of cranial and peripheral nerves and roots by lymphoma cells. ${ }^{[1]}$ We present the classical magnetic resonance imaging (MRI) findings of brachial plexopathy in a 15-year-old boy with Non-Hodgkin's Lymphoma (NHL) who presented with right arm weakness six months after complete remission.

MRI of the brachial plexus demonstrated thickening and hyperintensity of the C5 root and upper trunk of the right brachial plexus. There was also hyperintensity of the adjacent spinal cord [Figure 1A and B]. Post Gadolinium images showed significant contrast enhancement of the involved trunk, nerve root and the spinal cord at C4-5 level [Figures 2 and 3]. A concomitant CSF examination revealed the presence of multiple, abnormal B-cell lymphocytes. In view of these typical MRI and CSF findings, a diagnosis of B-cell neurolymphomatosis involving the right brachial plexus was made and the patient was started on salvage chemotherapy.

Patients of NL commonly present with a progressive sensorimotor peripheral neuropathy, plexopathy or cranial neuropathy. A histopathological demonstration of malignant lymphocytes in the peripheral nerves is

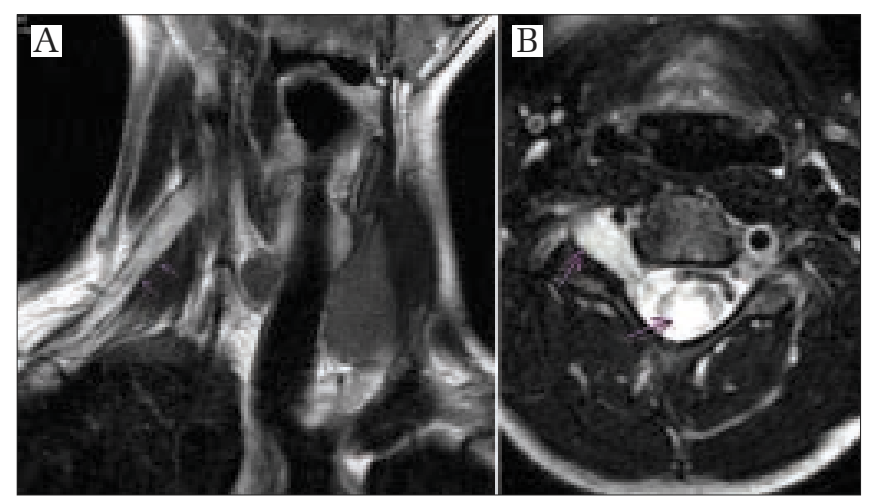

Figure 1: (A) T2W coronal oblique MR image of neck shows thickening and hyperintensity of the right brachial plexus (arrows), (B) T2W axial MR image at C 4-5 level shows thickening and hyperintensity of the right nerve root and the cord substance

\section{MRI diagnosis of neurolymphomatosis of the brachial plexus}

Sir,

Neurolymphomatosis (NL) is a rare manifestation of lymphoma which is characterized by selective

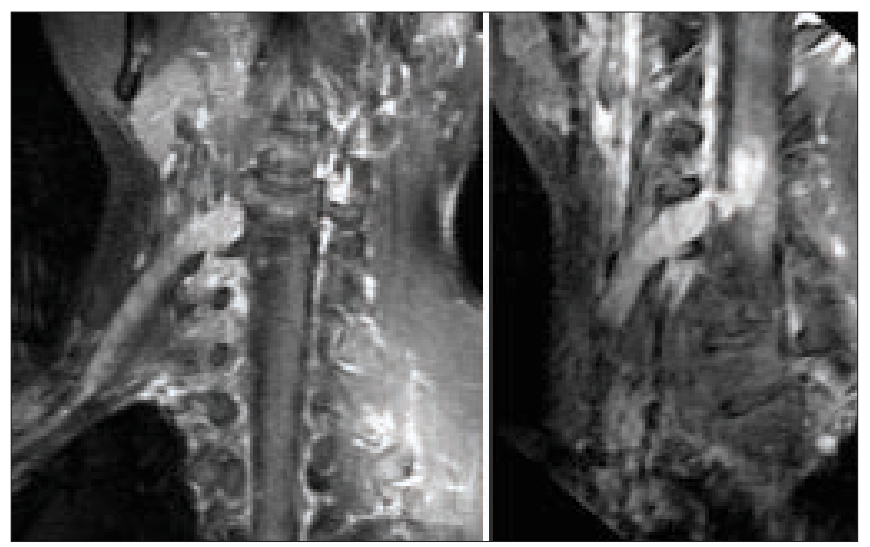

Figure 2: 3-D rendered images of a fat suppressed post gadolinium T1W coronal sequence show homogenous enhancement of the thickened right brachial plexus involving the trunks, C4-5 nerve root and the cord 


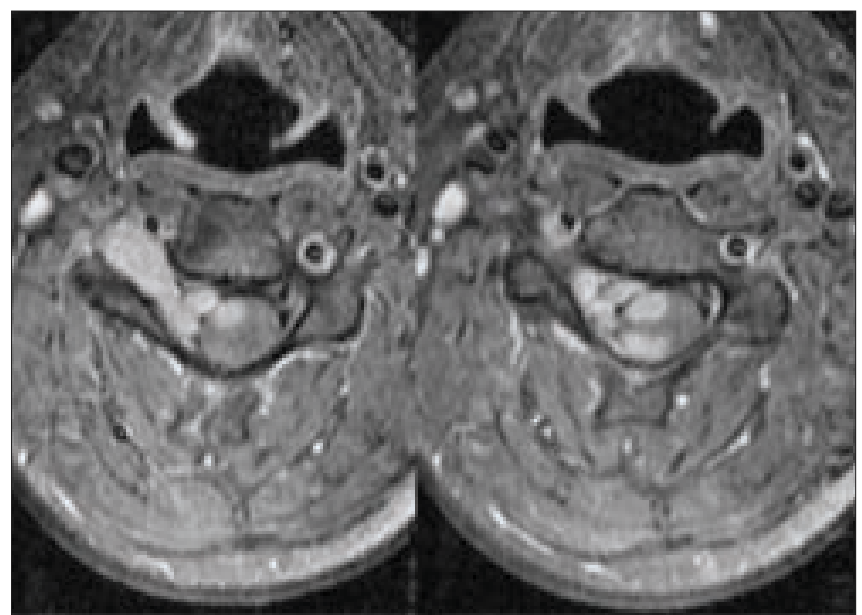

Figure 3: Axial fat suppressed post gadolinium T1W images at C4-5

level demonstrate florid enhancement of the nerve root in the right intervertebral foramen and further extension along the ventral and dorsal rami into the cord substance

the gold standard for diagnosis but a biopsy may not always be possible or positive. ${ }^{[2]}$ In such situations, MRI can be of immense diagnostic value by demonstrating diffuse neural thickening and enhancement and thus enabling an early diagnosis of NL. ${ }^{[1,3]}$

Arti Chaturvedi, J. P. Singh, Vikas Rastogi Department of Radiodiagnosis, Command Hospital Air Force, Bangalore, India. E-mail: docarti@gmail.com

\section{References}

1. Baehring JM, Damek D, Martin EC, Betensky RA, Hochberg FH. Neurolymphomatosis. Neuro Oncol 2003;5:104-15.

2. Shibata-Hamaguchi A, Samuraki M, Furui E, Ishida C, Kitagawa S, Nakao S, et al. B-cell neurolymphomatosis confined to the peripheral nervous system. J Neurol Sei 2007;260:249-52.

3. Swarnkar A, Fukui MB, Fink DJ, Rao GR. MR imaging of brachial plexopathy in neurolymphomatosis. AJR Am J Roentgenol 1997;169:1189-90.

Accepted on 05-09-2008 